\title{
Prickly waterlily and rigid hornwort genomes shed light on early angiosperm evolution
}

\author{
Yongzhi Yang1,2,7, Pengchuan Sun ${ }^{103,7}$, Leke Lv1, Donglei Wang1, Dafu Ru1, Ying Li², Tao Ma1, Lei Zhang1, \\ Xingxing Shen ${ }^{4}$, Fanbo Meng ${ }^{3}$, Beibei Jiao ${ }^{3}{ }^{3}$, Lanxing Shan ${ }^{3}$, Man Liư ${ }^{3}$ Qingfeng Wang ${ }^{5}$, Zhiji Qin ${ }^{3}$,

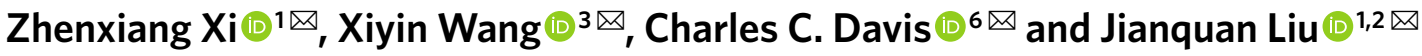

\begin{abstract}
Angiosperms represent one of the most spectacular terrestrial radiations on the planet ${ }^{1}$, but their early diversification and phylogenetic relationships remain uncertain ${ }^{2-5}$. A key reason for this impasse is the paucity of complete genomes representing early-diverging angiosperms. Here, we present high-quality, chromosomal-level genome assemblies of two aquatic species-prickly waterlily (Euryale ferox; Nymphaeales) and the rigid hornwort (Ceratophyllum demersum; Ceratophyllales)-and expand the genomic representation for key sectors of the angiosperm tree of life. We identify multiple independent polyploidization events in each of the five major clades (that is, Nymphaeales, magnoliids, monocots, Ceratophyllales and eudicots). Furthermore, our phylogenomic analyses, which spanned multiple datasets and diverse methods, confirm that Amborella and Nymphaeales are successively sister to all other angiosperms. Furthermore, these genomes help to elucidate relationships among the major subclades within Mesangiospermae, which contain about 350,000 species. In particular, the species-poor lineage Ceratophyllales is supported as sister to eudicots, and monocots and magnoliids are placed as successively sister to Ceratophyllales and eudicots. Finally, our analyses indicate that incomplete lineage sorting may account for the incongruent phylogenetic placement of magnoliids between nuclear and plastid genomes.
\end{abstract}

The angiosperms, or flowering plants, represent one of the most diverse and species-rich clades on Earth. They provide the vast majority of food consumed by humans and contribute substantially to global photosynthesis and carbon sequestration ${ }^{1}$. The origin of angiosperms was famously coined 'an abominable mystery' owing to their sudden appearance and rapid diversification ${ }^{2-5}$. To date, angiosperms include more than 350,000 species $^{6}$ and occupy nearly every habitat from forests and grasslands to sea margins and deserts; angiosperms encompass a considerable variety of life forms, including trees, herbs, submerged aquatics and epiphytes. Resolving early angiosperm phylogeny is therefore critical for our understanding of such diversifying processes ${ }^{1,7}$.

Decades of efforts have greatly resolved the angiosperm phylogeny, illuminating their evolutionary history and helping to delineate major groups ${ }^{2-5}$. It has been identified that the three early-diverging angiosperm orders Amborellales, Nymphaeales and Austrobaileyales, which constitute remarkable morphological disparity and low species diversity, represent the earliest diverged angiosperm lineages ${ }^{8}$ (that is, the so-called ANA grade). However, the vast majority of angiosperms belong to the Mesangiospermae clade, which includes approximately $99 \%$ of all extant angiosperms. Eudicots and monocots are the two largest Mesangiospermae subclades, including around $75 \%$ and $22 \%$ of all species, respectively'; magnoliids represent a third subclade with about 9,000 species $^{10}$; and the remaining two subclades, Chloranthales and Ceratophyllales, are morphologically unusual with only 77 and 7 species, respectively ${ }^{10-12}$. Despite the elucidation and the strong support for each of the five subclades of Mesangiosperms ${ }^{4,13}$, phylogenetic relationships among these clades remain uncertain, and different topologies have been proposed on the basis of various morphological ${ }^{14}$ and/or molecular lines of evidence ${ }^{13,15-18}$ (Supplementary Fig. 1).

Genomic data provide a rich and convincing means to resolve such evolutionary uncertainties. Despite the availability of numerous sequenced genomes from eudicots and monocots, early-diverging angiosperms remain poorly sampled, therefore inhibiting insights into these fundamental questions. To date, no nuclear genome has been sequenced for the four key orders-Austrobaileyales, Ceratophyllales, Chloranthales and Nymphaeales-which exhibit diverse life histories, extreme morphological variation and great evolutionary divergence. This lack of critical taxon sampling probably exacerbates phylogenetic uncertainty when inferring early angiosperm relationships. For example, nuclear genomes of three magnoliids (that is, Cinnamomum kanehirae, Liriodendron chinense and Persea americana) have been subsequently published ${ }^{19-21}$; however, phylogenetic analyses in these two studies resulted in conflicting placement of magnoliids relative to monocots and eudicots-that is, either monocots as the sister to a clade of magnoliids and eudicots, or magnoliids as the sister to monocots and eudicots ${ }^{19-22}$. Moreover, cases of deep phylogenetic incongruence between nuclear and organellar genomes have been recently reported in angiosperms ${ }^{18-24}$, but their causation (such as hybridization and incomplete lineage sorting (ILS)) has not been fully evaluated.

Here we report the high-quality chromosomal-level genome assemblies of E. ferox Salisb. (prickly waterlily; estimated genome size of $768.2 \mathrm{Mb}$ ) and C. demersum L. (rigid hornwort; estimated

${ }^{1}$ Key Laboratory of Bio-Resource and Eco-Environment of Ministry of Education \& State Key Laboratory of Hydraulics \& Mountain River Engineering, College of Life Sciences, Sichuan University, Chengdu, China. ${ }^{2}$ State Key Laboratory of Grassland Agro-Ecosystem, Institute of Innovation Ecology, Lanzhou University, Lanzhou, China. ${ }^{3}$ School of Life Sciences, North China University of Science and Technology, Tangshan, China. ${ }^{4}$ Institute of Insect Sciences, College of Agriculture and Biotechnology, Zhejiang University, Hangzhou, China. ${ }^{5}$ Key Laboratory of Aquatic Botany and Watershed Ecology, Wuhan Botanical Garden, Chinese Academy of Sciences, Wuhan, China. ${ }^{6}$ Department of Organismic and Evolutionary Biology, Harvard University Herbaria, Cambridge, MA, USA. ${ }^{7}$ These authors contributed equally: Yongzhi Yang, Pengchuan Sun. ${ }^{-}$e-mail: zxi@scu.edu.cn; wangxiyin@vip.sina.com; 
genome size of $777.2 \mathrm{Mb}$ ), which are representatives of the two aquatic lineages Nymphaeales and Ceratophyllales, respectively (Supplementary Fig. 2, Supplementary Table 1). A total of $31.7 \mathrm{~Gb}$ of Oxford Nanopore Technologies (ONT) long reads and $47.4 \mathrm{~Gb}$ of Illumina short reads were generated for Euryale, and $80.5 \mathrm{~Gb}$ of ONT long reads and $46.4 \mathrm{~Gb}$ of short reads were generated for Ceratophyllum (Supplementary Fig. 3, Supplementary Table 2). ONT long reads were de novo assembled into contigs using the Canu assembler ${ }^{25}$, and two rounds of polishing were applied to the assembled contigs using Pilon ${ }^{26}$ with the Illumina short reads. The resulting genome assemblies of Euryale and Ceratophyllum were $725.2 \mathrm{Mb}$ (N50 size of $4.75 \mathrm{Mb}$, where N50 corresponds to the minimum contig length needed to cover $50 \%$ of the genome) and $733.3 \mathrm{Mb}$ (N50 size of $1.56 \mathrm{Mb}$ ), respectively (Supplementary Table 3). Moreover, a total of $84.4 \mathrm{~Gb}$ and $133.9 \mathrm{~Gb}$ of Hi-C data were generated using the Illumina platform for Euryale and Ceratophyllum, respectively. Assembled contigs were then clustered into 29 and 12 pseudochromosomes for Euryale and Ceratophyllum, respectively, using LACHESIS $^{27}$ (Fig. 1a, Supplementary Tables 2 and 4). Both genome assemblies showed a high contiguity, completeness and accuracy (Fig. 1a, Supplementary Fig. 4, Supplementary Tables 5-7), and matched the chromosome counts obtained from cytological studies $^{28}$. Using a combination of homology-based and transcriptomebased approaches, 40,297 and 30,138 protein-coding genes were predicted in the genomes of Euryale and Ceratophyllum, respectively (Supplementary Fig. 5, Supplementary Table 8). Moreover, $78.3 \%$ and $71.2 \%$ of all of the predicted protein-coding genes were clustered into gene families for Euryale and Ceratophyllum, respectively (Supplementary Note 1, Supplementary Fig. 6, Supplementary Table 9), and $85.6 \%$ and $89.8 \%$ of all of the predicted proteincoding genes were successfully annotated by at least one database (that is, SwissProt, TrEMBL, InterPro, GO or KEGG) for Euryale and Ceratophyllum, respectively (Supplementary Table 10). Furthermore, despite the similar genome size of these two species, the percentage of predicted repetitive elements was much higher in the genome of Ceratophyllum (that is, $38.35 \%$ versus $63.08 \%$ for Euryale and Ceratophyllum, respectively; Supplementary Table 11).

By constructing the distribution of synonymous substitutions per synonymous site (Ks) using syntenic paralogues within each genome, we detected two and three polyploidization events in the genomes of Euryale and Ceratophyllum, respectively (Supplementary Fig. 7, Supplementary Table 12). After correction for evolutionary rate $^{29}$, the two polyploidization events in the genome of Euryale were estimated to occur at approximately 16-18 million and 94-106 million years ago (Ma), respectively; the three polyploidization events in Ceratophyllum were estimated to occur approximately 13-15 Ma, 127-143 Ma and 157-177 Ma, respectively (Fig. 1b). Furthermore, we identified polyploidization events in the genomes of C. kanehirae, P. americana, L. chinense, Oryza sativa and Vitis vinifera. Interestingly, the Cinnamomum and Persea genomes share two recent polyploidization events, and multiple independent polyploidization events have occurred in each of five major clades (that is, Nymphaeales, magnoliids, monocots, Ceratophyllales and eudicots; Fig. 1b, Supplementary Fig. 7), paralleling recent studies demonstrating that whole-genome duplication (WGD) is a widespread and potentially important evolutionary feature in angiosperms $s^{30,31}$. To better elucidate the polyploidy of our newly assembled genomes, we conducted a more focused comparative genomic analysis using Amborella, Cinnamomum, Liriodendron and Vitis as placeholders. Syntenic depth ratios of 6:1, 6:4, 6:2 and 6:3 were inferred in the Euryale-Amborella, Euryale-Cinnamomum, Euryale-Liriodendron and Euryale-Vitis comparisons, respectively, and 8:1, 8:4, 8:2 and 8:3 in the Ceratophyllum-Amborella, Ceratophyllum-Cinnamomum, Ceratophyllum-Liriodendron and Ceratophyllum-Vitis comparisons, respectively (Fig. 1c, Supplementary Figs. 8 and 9). On the basis of the syntenic relationships between and within each species, our analyses collectively demonstrate that Euryale underwent an ancient WGD followed by one whole-genome triplication, and Ceratophyllum has undergone three WGDs.

For the first time, the genomic taxon sampling represents two of the three orders in the ANA grade and four of the five subclades of Mesangiospermae. To resolve early angiosperm phylogeny, a total of 1,374 single-copy nuclear genes (SSCGs) were first identified with SonicParanoid ${ }^{32}$ using whole-genome sequences from 14 seed plants-that is, four eudicots (Aquilegia coerulea, Arabidopsis thaliana, Prunus persica and Vitis), three monocots (Musa acuminate, Oryza and Phalaenopsis equestris), three magnoliids (Cinnamomum, Liriodendron and Persea), Ceratophyllum, two ANA-grade species (Amborella and Euryale) and one gymnosperm (Ginkgo biloba; Supplementary Table 13). Aligned protein-coding regions were concatenated and analysed using two methods-(1) including all three codon positions (SSCG-CDS) and (2) including only the first and second codon positions (SSCG-Codon12). Moreover, for coalescent-based analyses, gene trees were individually estimated from each of the two datasets (SSCG-CDS and SSCG-Codon12), which were then input into ASTRAL ${ }^{33}$ for species tree inference (Supplementary Fig. 10). Our estimated gene trees are generally well supported (Supplementary Fig. 11), and both concatenation and coalescent analyses produced an identical strongly supported topology (Fig. 2a,b, Supplementary Figs. 12-14). Here, Amborella and Euryale were placed as successively sister to all other angiosperms, and monocots and magnoliids were inferred as successively sister to Ceratophyllum and eudicots. Our phylogenetic placement of magnoliids differs from APG, but is consistent with other studies that used various molecular markers, such as the plastid inverted repeat region ${ }^{34}$ and transcriptome data ${ }^{18,35}$. To avoid potential errors in orthology inference, we also extracted single-copy genes using OrthoMCL ${ }^{36}$ from the 14 seed plants described above as well as another gymnosperm (Picea abies). Only those genes sampled from at least 11 species were selected for downstream analyses, and a total of 2,302 single-copy genes (OSCG) were retained with an average of 1,859 genes for each species. Concatenation and coalescent analyses were similarly conducted as got those described above, and corroborated our phylogenetic findings (Fig. 2a, Supplementary Fig. 15). Furthermore, we took advantage of the newly developed species-tree inference method $\mathrm{STAG}^{37}$, which was designed to leverage gene trees estimated from multi-copy gene families. Only those genes sampled from all 15 seed plants were included, and a total of 2,356 low-copy genes (LCG) were retained. Species trees inferred from datasets including all three codon positions (LCG-CDS) and the first and second codon positions only (LCG-Codon12) were topologically identical to the ones described above (Fig. 2a, Supplementary Fig. 16), suggesting that our findings are robust.

Despite the fact that the same set of phylogenetic relationships was consistently recovered when nuclear genes were analysed simultaneously, topological conflicts among gene trees were widely observed as visualized using DensiTree ${ }^{38}$ (Fig. 2c). A major discordance was identified in the datasets SSCG-CDS and SSCG-Codon12 involving the relationship between Amborella and Nymphaeales (Fig. 2c). For the datasets SSCG-CDS and SSCG-Codon $12,46.3 \%$ and $44.5 \%$ of all 1,374 gene trees supported Amborella and Euryale as successively sister to all other angiosperms, respectively; $27.2 \%$ and $26.0 \%$ supported Amborella as sister to Euryale, respectively; and the other 26.5\% and 29.5\% supported Euryale alone as the first lineage of angiosperms, respectively (Fig. 2d, Supplementary Figs. 17-19). We also summarized gene tree discordance using DiscoVista ${ }^{39}$, and similar results were observed for the datasets SSCG-CDS and SSCG-Codon12-that is, a substantial fraction of gene trees were incongruent with species trees regarding the placement of Amborella and Nymphaeales (Fig. 2e, Supplementary Figs. 17-19). Moreover, conflicting phylogenetic placements of Ceratophyllales were observed in the gene trees. For the datasets SSCG-CDS and 
a

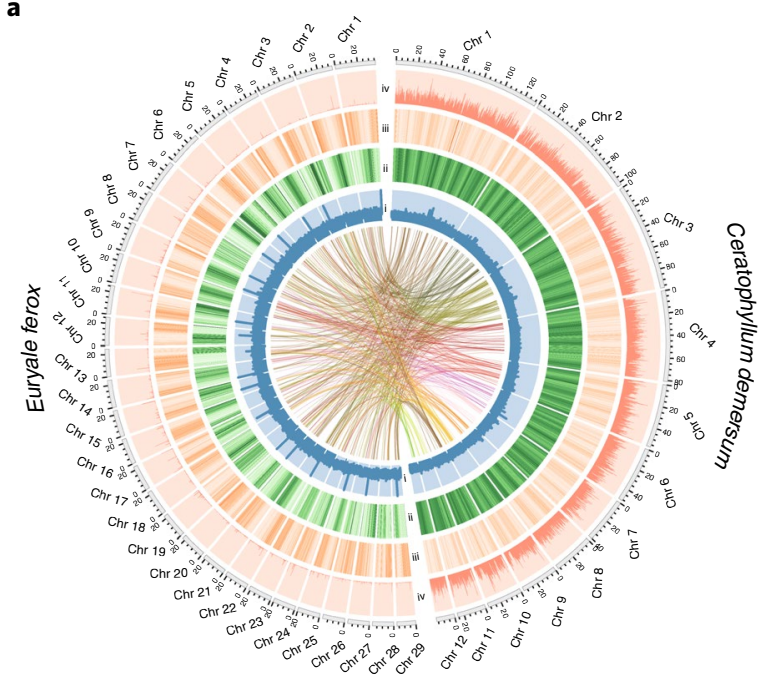

b

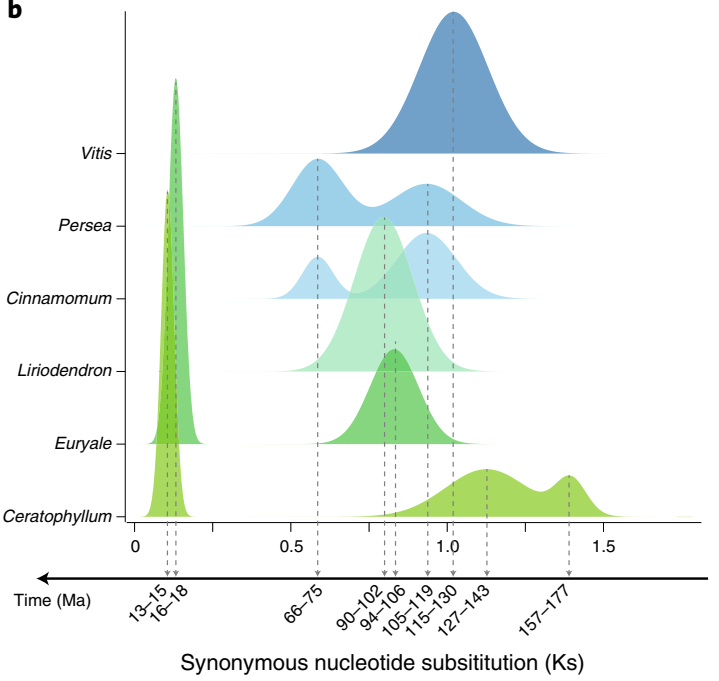

c
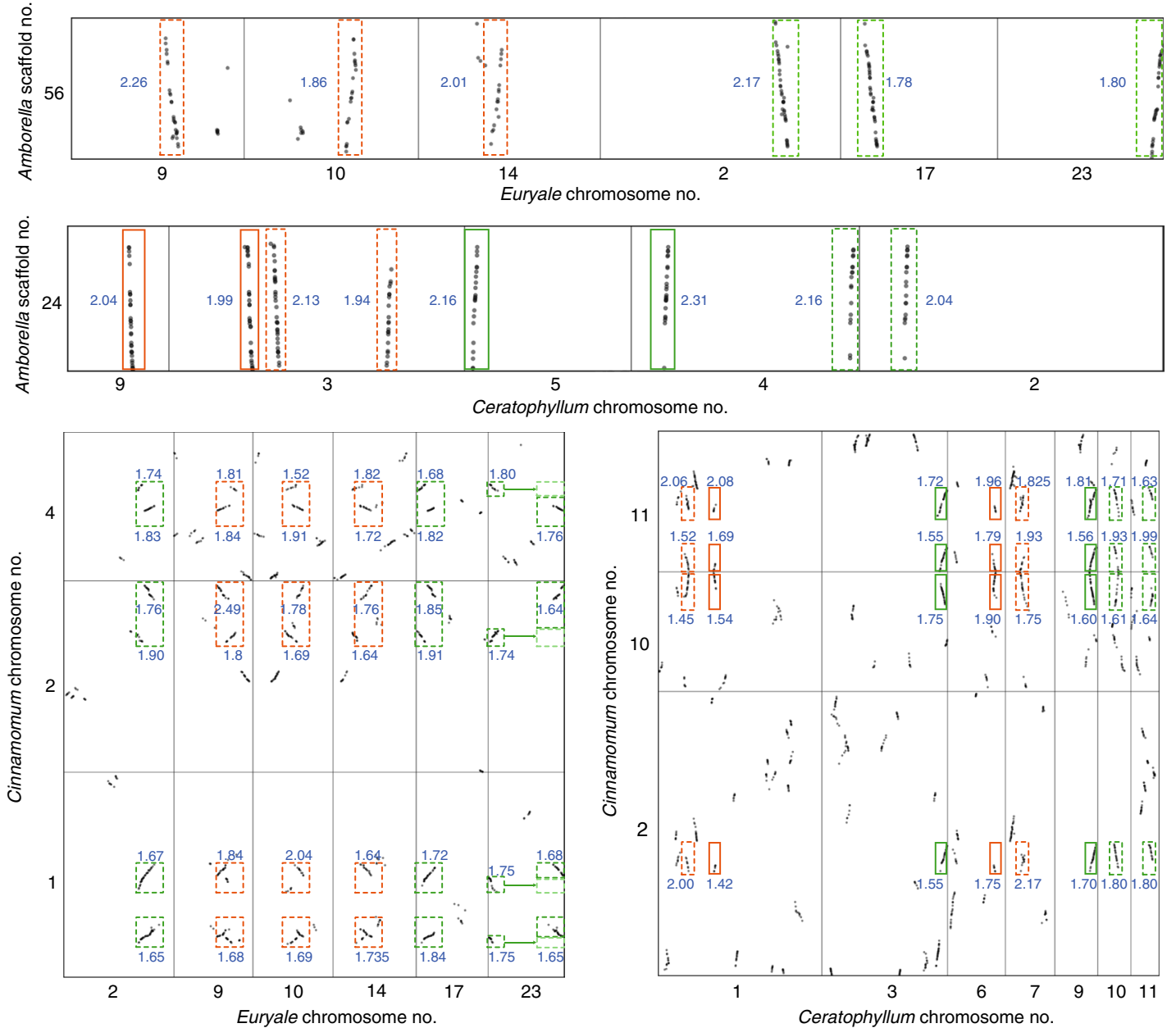

Fig. 1 C Comparative genomics analyses. a, The genomic features of $E$. ferox (pseudomolecules size: $721.2 \mathrm{Mb}$ ) and $C$. demersum (pseudomolecules size: 703.8 Mb). From inside to outside: GC content in 500-bp sliding windows (i; minimum-maximum, 0.2-0.8); repeat density in 10-kb sliding windows (ii; minimum-maximum: 0-1.0, coloured from white to dark green); gene density in 100-kb sliding windows (iii; minimum-maximum, 0-30, coloured from white to dark orange); and SNV density in 100-kb sliding windows (iv; minimum-maximum: 0-0.025). The links in the centre connect syntenic gene blocks that were detected using MCscan. Chr, chromosome. b. Distribution of average synonymous substitution levels (Ks) between syntenic blocks after evolutionary rate correction. c, Syntenic blocks (involving $\geq 10$ colinear genes) between genomes. The corresponding median Ks value is shown for each block, and polyploidization events are represented by different colours.

SSCG-Codon $12,39.7 \%$ and $34.2 \%$ of 1,374 gene trees supported Ceratophyllum as sister to eudicots, respectively; $27.6 \%$ and $32.9 \%$ supported Ceratophyllum as sister to monocots, respectively; and the other $32.7 \%$ and $32.9 \%$ supported Ceratophyllum as sister to magnoliids, respectively (Fig. 2d, Supplementary Figs. 17-19). These analyses indicate that there is probably substantial ILS during 
a

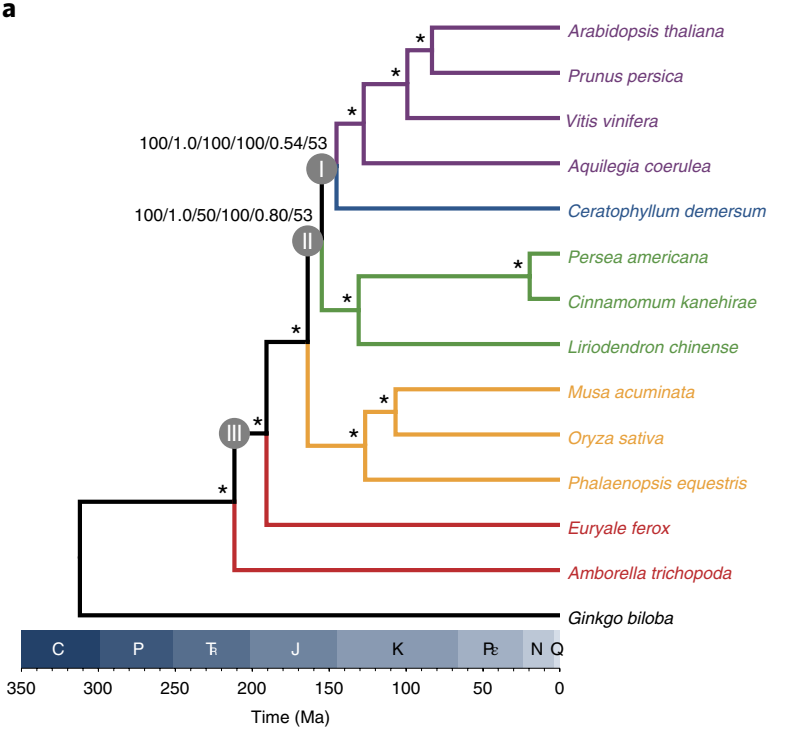

c

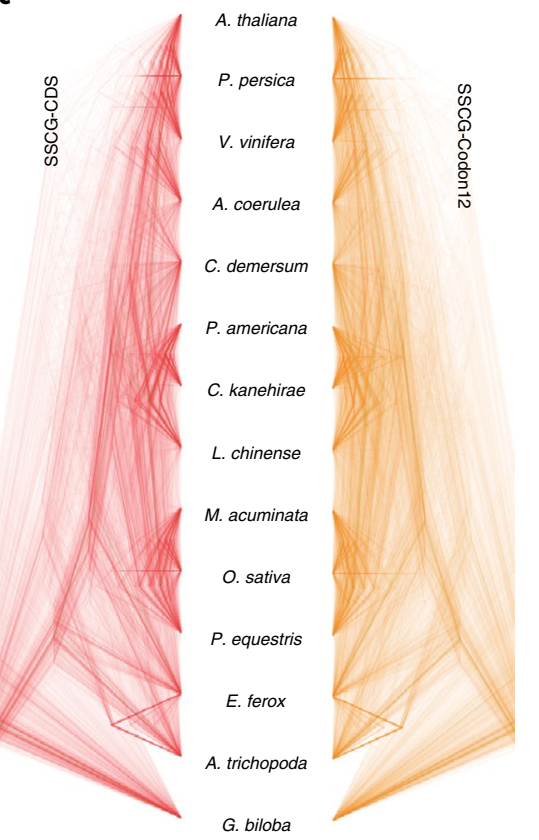

d
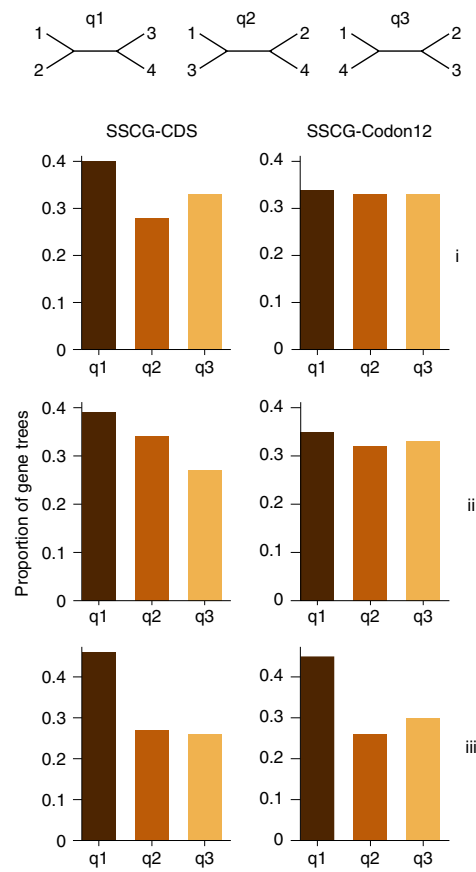

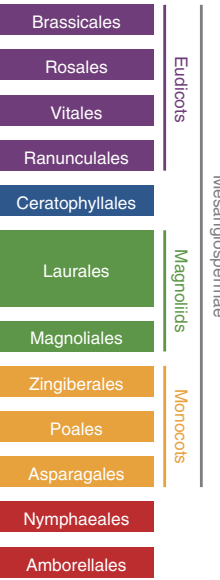

b

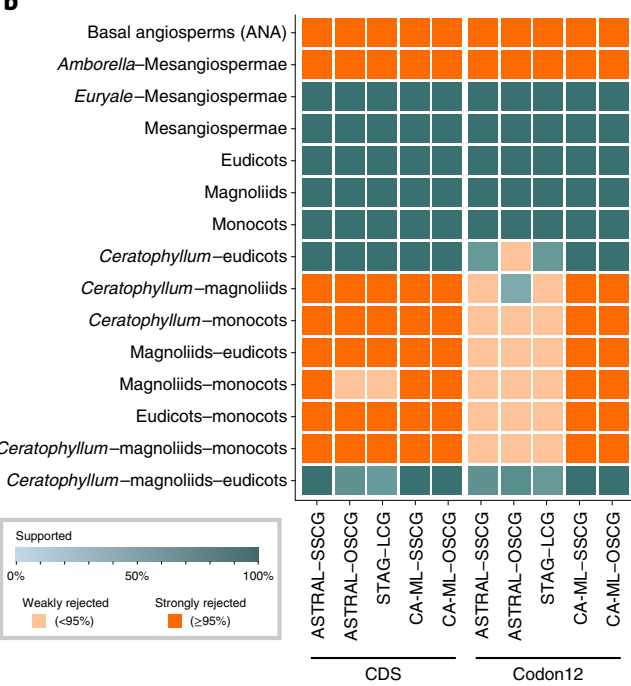

e

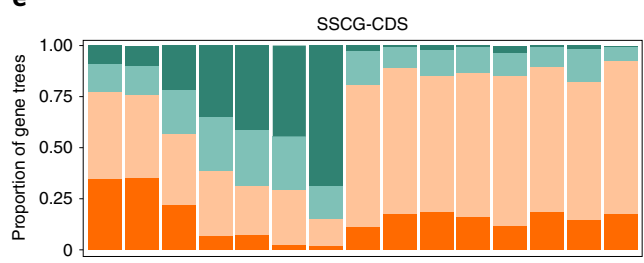

SSCG-Codon12
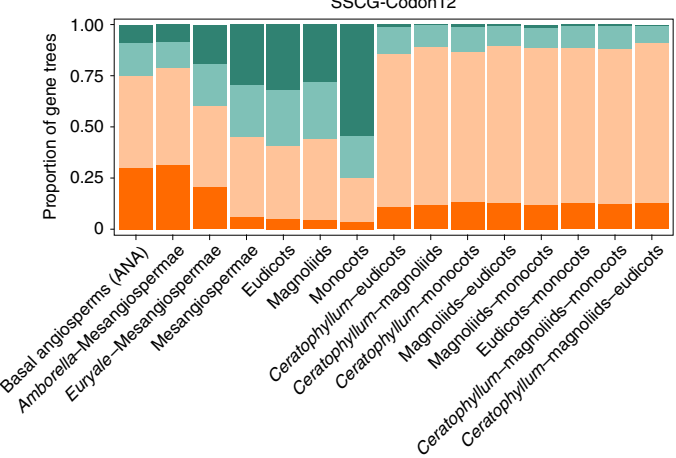

Strongly supported Weakly supported Weakly rejected Strongly rejected

Fig. 2 | Phylogenomic analyses of early-diverging angiosperms. a, Chronogram of early-diverging angiosperms on the basis of the dataset SSCG-CDS inferred using MCMCTree. Bootstrap support percentages and posterior probabilities are indicated for each internal branch (from left to right, SSCG-CDS concatenation analysis using maximum likelihood (CA-ML), SSCG-CDS ASTRAL, LCG-CDS STAG, SSCG-Codon12 CA-ML, SSCG-Codon12 ASTRAL, LCGCodon12 STAG), and an asterisk indicates 100 bootstrap support percentages and 1.0 posterior probabilities in all analyses. i, ii and iii indicate each internal branch. C, Carboniferous; J, Jurassic; K, Cretaceous; N, Neogene; P, Permian; $\nwarrow$, Triassic; Q, Quaternary; Pe , Palaeogene. b, Species tree analysis using DiscoVista. Rows correspond to focal splits, and the spectrum indicates the support value for splits that are compatible with a species tree. Teal indicates themonophyly of a clade, and the different shades of teal indicate the level of its bootstrap support percentage (0 to $100 \%)$. Orange indicates rejection of a clade, and a 95\% cut-off (instead of astandard 75\%) was selected for strong rejection due to higher support values with genome-scale data. c, Superimposed ultrametric gene trees in a consensus DensiTree plot. The datasets SSCG-CDS and SSCG-Codon12 are shown in red and orange, respectively. $\mathbf{d}$, The frequency of three topologies (q1-q3) around focal internal branches of ASTRAL species trees in the datasets SSCG-CDS and SSCGCodon12. Each internal branch (labelled i, ii and iii) with four neighbouring branches can lead to three possible topologies (for example, q1, q2 and q3). e, Gene tree compatibility. The portion of gene trees for which focal splits are highly (or weakly) supported (or rejected). Weakly rejected splits are those that are not in the tree but are compatible if low support branches (below 75\%) are contracted.

early angiosperm evolution and greatly highlight the phylogenomic complexity of resolving early-diverging angiosperms.

Furthermore, phylogenetic analyses of these 15 seed plants inferred from 72 concatenated plastid genes strongly support magnoliids as the first diverging lineage of Mesangiospermae (Supplementary Fig. 20, Supplementary Table 14). This placement of magnoliids is incongruent with our nuclear phylogeny, but consistent with a recent study that analysed 2,881 plastomes ${ }^{4}$. 
Thus, what might account for this deep phylogenetic incongruence between nuclear and plastid genomes? As multiple independent polyploidization events were identified in magnoliids, monocots, Ceratophyllales and eudicots (Fig. 1), allopolyploidization or hybridization is one probable source of genomic discordance. We first assessed putative hybridization events in our phylogeny using PhyloNetworks. Although three cases of hybridization were inferred, none involved the three species of magnoliids (that is, Cinnamomum, Liriodendron and Persea; Supplementary Fig. 21). Furthermore, very short internal branches among four subclades of Mesangiospermae were observed in all our analyses, corresponding to an estimated divergence time of around $20 \mathrm{Ma}$ (Supplementary Figs. 12-16, 20 and 22). We therefore tested whether ILS might better explain this discordance. We simulated 20,000 gene trees under the multispecies coalescent model ${ }^{40}$ on the basis of the ASTRAL tree inferred from the dataset SSCG-CDS. We found considerable agreement between simulated and empirical gene trees (overall correlation coefficient, Spearman's $\rho=0.97, P<0.01$; Supplementary Fig. 23), suggesting that the multispecies coalescent model is a good fit to our data. Here the relative frequencies of various topologies, including the topology inferred from plastomes, were consistent with frequencies of ILS as estimated from our coalescent analyses (Supplementary Fig. 23c,d). These results indicate that ILS may well account for the incongruent placement of magnoliids between nuclear and plastid genomes. Finally, as sparse taxon sampling could result in these discordant results ${ }^{41}$, we increased our taxon sampling in the nuclear phylogeny by adding taxa with published genomes. A total of 612 'mostly' single-copy orthologous genes (SCOG) were extracted from 213 nuclear genomes, which included 211 angiosperms representing 33 orders and 67 families as well as two gymnosperms as outgroups (Supplementary Table 15), and the average number of genes per taxon was 545 . Coalescent analyses of the datasets SCOG-CDS and SCOG-Codon 12 recovered the same relationships among the four subclades of Mesangiospermae (Supplementary Figs. 24-26), suggesting that our results are robust to additional taxa sampling.

In summary, the high-quality genomes of prickly waterlily and rigid hornwort greatly help to clarify phylogenetic relationships of early-diverging angiosperms. Moreover, these genomic resources are essential for future comparative investigations of genic evolution that underpin the morphological, physiological and ecological diversification of early angiosperms (Supplementary Notes 1-3, Supplementary Figs. 27-28, Supplementary Tables 16-22).

\section{Methods}

Plant materials and DNA sequencing. Fresh leaves of E. ferox and whole plants of $C$. demersum were obtained for DNA extraction and sequencing. Total genomic DNA was extracted using the CTAB method ${ }^{42}$. The library for ONT sequencing was constructed using large $(>20 \mathrm{~kb}$ ) DNA fragments with the Ligation Sequencing Kit 1D (SQK-LSK108), and sequenced using the GridION X5 platform. Adapters and low-quality nucleotides (that is, mean quality score $<7$ ) were trimmed. Pairedend libraries with an insertion size of $350 \mathrm{bp}$ were constructed according to the manufacturer's protocols and sequenced using the Illumina HiSeq 2500 System. Illumina reads were filtered using following criteria: (1) containing more than $5 \%$ unidentified nucleotides, (2) more than $65 \%$ of bases with a Phred quality score $<7$ and (3) more than $10 \mathrm{bp}$ adapter sequences (allowing $2 \mathrm{bp}$ mismatches). For the high-throughput chromosome conformation capture (Hi-C) analysis, fresh leaves were fixed in formaldehyde solution (1\%), and chromatin was cross-linked and digested using the restriction enzyme HindIII. The $5^{\prime}$ overhangs were filled-in with biotinylated nucleotides, and free blunt ends were ligated. After ligation, crosslinks were reversed and the DNA was purified to remove protein. Purified DNA was treated to remove biotin that was not internal to ligated fragments. DNA was sheared into fragments of $\sim 350 \mathrm{bp}$, and sequenced using the Illumina platform.

Genome size estimation. Genome size was estimated using the $k$-mer analysis of Illumina 150 -bp paired-end reads. The $k$-mer depth-frequency distribution was generated using SOAPec ${ }^{43}$ (v.2.0.1, https://sourceforge.net/projects/soapdenovo2/) with the following parameters: -k $17-\mathrm{q} 33-\mathrm{t} 10$. The genome size was then calculated according to the following formula ${ }^{44}$ : genome size $=k$-mer coverage/ mean $k$-mer depth (Supplementary Table 1).
Genome assembly. ONT long reads were de novo assembled using the Canu $\operatorname{assembler}^{25}$ (v.1.7, https://github.com/marbl/canu/), and two rounds of polishing were applied to the assembled contigs using Pilon $^{26}$ (v.1.22, https://github.com/ broadinstitute/pilon/) with the Illumina short reads. HiC-Pro ${ }^{45}$ v.2.10.0 was used to evaluate the quality of $\mathrm{Hi}-\mathrm{C}$ data. Valid interaction pairs were mapped to the contigs and anchored to the pseudochromosomes using LACHESIS ${ }^{27}$ (https:// github.com/shendurelab/LACHESIS).

Transcriptome sequencing and assembly. For each species, total RNA was extracted from various plant organs (roots, leaves and stems), and residual DNA was removed using the DNA-free DNA Removal Kit. A total of $18.69 \mathrm{~Gb}$ and $5.85 \mathrm{~Gb}$ of reads were generated using the Illumina platform for E. ferox and C. demersum, respectively. Transcripts were assembled from filtered reads using Trinity ${ }^{46}$ v.2.8.4 with additional parameters including '--trimmomatic --normalize_reads'.

Annotation of repetitive elements. To annotate repetitive elements, we utilized a combination of evidence-based and de novo approaches. Genome assemblies were first searched using RepeatMasker ${ }^{47}$ (v.4.0.7, http://repeatmasker.org/) against the Repbase database (http://www.girinst.org/repbase). Next, a de novo repetitive-element library was constructed using RepeatModeler (v.1.0.11, http:// repeatmasker.org/RepeatModeler.html). This de novo repetitive-element library was then utilized by RepeatMasker to annotate repetitive elements. Results from these two runs of RepeatMasker were merged.

Protein-coding gene prediction and functional annotation. The identification of protein-coding genes was based on transcriptome data and ab initio prediction. RNA transcripts were first mapped to the assembled genome using PASA ${ }^{48}$ (Program to Assemble Spliced Alignment v.2.3.3). Valid transcript alignments were clustered on the basis of mapping location and assembled into gene structures, and then the high-quality gene models were selected for training by AUGUSTUS ${ }^{49}$ v.3.2.3. Moreover, intron hints were generated using the script bam2hints provided by AUGUSTUS. Next, AUGUSTUS was utilized for ab initio gene prediction on the hard-masked genome assembly, and all of the predictions were integrated using EvidenceModeler ${ }^{49}$ (EVM, v.1.1.1) to generate consensus gene sets. For functional annotation, our predicted protein-coding genes were searched against the SwissProt and TrEMBL databases, as well as the InterPro database using InterProScan ${ }^{50}$ release $5.33-72.0$.

Polyploidization analysis. Seven genomes were selected for our polyploidization analysis, that is, A. trichopoda (Amborellales; At), C. demersum (Ceratophyllales), C. kanehirae (magnoliids), E. ferox (Nymphaeales; Ef), L. chinense (magnoliids), $O$. sativa (monocots), P. Americana (magnoliids) and V. vinifera (eudicots; Vv). Colinear genes within each genome and between genomes were inferred using MCScan ${ }^{51}$ v.0.8 according to the combined information of gene similarity and gene order. Synonymous substitutions per synonymous site (Ks) between colinear genes were estimated using the Nei-Gojobori approach ${ }^{52}$ as implemented in the PAML $^{53}$ package v.4.9h. The median Ks values were selected to represent each syntenic block, and the probability density distribution curve of Ks was estimated using MATLAB with the kernel smoothing density function (ksdensity; bandwidth was typically set to 0.025 ). Multipeak fitting of the curve was performed using the Gaussian approximation function (cftool) in MATLAB, and the coefficient of determination $\left(R^{2}\right)$ was set as at least 0.95 .

Furthermore, we performed a correction to the Ks values to distinguish the order of each polyploidization event using a similar method to a method used previously ${ }^{54}$. Here, supposing that the Ks values of colinear orthologues between two genomes $i$ and $j$ are $X_{i-j}: N\left(\mu_{i-j}, \sigma_{i-j}^{2}\right)$, where $N$ represents the normal distribution, $\mu$ represents the mean value and $\sigma$ represents the standard deviation. We further supposing that the ratio of the evolutionary rate of species $i$ to the assumed averaged evolutionary rate of angiosperms is $r_{i}$, the correction coefficient $\lambda_{i}$ is defined as $\lambda_{i}=\frac{1}{r_{i}}$ and, accordingly, the correction coefficient factor of $X_{i-j}$ is defined as $\lambda_{i j}=\lambda_{i} \lambda_{j}$

The mean of the corrected $X_{i-j \text { correction }}$ can be inferred to be

$$
\mu_{\mathrm{i}-\mathrm{j}-\text { correction }}=\mu_{i-j} \lambda_{i} \lambda_{j}
$$

For $E[t X]=t E[X]$ and $D[t X]=t^{2} D[X]$

we can get

$$
X_{i-j-\text { correction }}: N\left(\mu_{i-j-\text { correction }}, \sigma_{i-j-\text { correction }}^{2}\right)=N\left(\lambda_{i} \lambda_{j} \mu_{i-j}, \lambda_{i}^{2} \lambda_{j}^{2} \sigma_{i-j}^{2}\right)
$$

As Amborella and Euryale are basal angiosperms, the divergence between Amborella or Euryale and other plants occurred at the same time. Therefore, for genome $i$

$$
\begin{aligned}
& \frac{\mu_{\mathrm{At}-i-\text { correction }}}{\mu_{\mathrm{At}-\mathrm{Vv}-\text { correction }}}=\frac{\mu_{\mathrm{At}-i} \lambda_{\mathrm{At}} \lambda_{i}}{\mu_{\mathrm{At}-\mathrm{Vv}} \lambda_{\mathrm{At}} \lambda_{\mathrm{Vv}}}=\frac{\mu_{\mathrm{At}-i} \lambda_{i}}{\mu_{\mathrm{At}-\mathrm{Vv}} \lambda_{\mathrm{Vv}}}=1 \\
& \frac{\mu_{\mathrm{Ef}-i-\text { correction }}}{\mu_{\mathrm{Ef}-\mathrm{Vv}-\text { correction }}}=\frac{\mu_{\mathrm{Ef}-i} \lambda_{\mathrm{Ef}} \lambda_{i}}{\mu_{\mathrm{Ef}-\mathrm{Vv}} \lambda_{\mathrm{Ef}} \lambda_{\mathrm{Vv}}}=\frac{\mu_{\mathrm{Ef}-i} \lambda_{i}}{\mu_{\mathrm{Ef}-\mathrm{Vv}} \lambda_{\mathrm{Vv}}}=1
\end{aligned}
$$




$$
\frac{\lambda_{i}}{\lambda_{\mathrm{Vv}}}=a_{i}=\operatorname{mean}\left\{\frac{\mu_{\mathrm{At}-\mathrm{Vv}}}{\mu_{\mathrm{At}-i}}, \frac{\mu_{\mathrm{Ef}-\mathrm{Vv}}}{\mu_{\mathrm{Ef}-i}}\right\}
$$

$$
\lambda_{i}=\lambda_{\mathrm{Vv}} a_{i}
$$

The $a_{i}$ represents the mean ratio value among the observed Ks peak between Amboralla and Vitis, or Euryale and Vitis. After the divergence from the other studied plants, $A$. trichopoda has not been affected by polyploidization anymore; thus, we assumed that the evolutionary rate of Amborella genes is relatively stable and, therefore, set $\lambda_{\mathrm{At}}=1$. The plant $i$ with the slowest evolutionary rate is the most likely to have the same evolutionary rate as Amborella, that is, $\max \left\{\lambda_{i}\right\}=1$ and $\lambda_{\mathrm{Vv}}=\frac{\max \left\{\lambda_{i}\right\}}{\max \left\{a_{i}\right\}}=\frac{1}{\max \left\{a_{i}\right\}}$. We determined the approximate value for $\operatorname{V}$. vinifera $\left(\lambda_{\mathrm{Vv}}\right)$ using the above estimator, and used it to assess the correction coefficient ratio for each species. The major-eudicot common hexaploidy $115-130 \mathrm{Ma}$ (refs. ${ }^{55,56}$ ), inferred by grape duplicated genes, was used as the reference to date the ages for the other polyploidization and speciation events (Supplementary Table 12).

Phylogenetic analyses. To infer the phylogenetic placements of E. ferox and $C$ demersum, SSCGs were first identified using SonicParanoid ${ }^{32}$ v.1.0 from 14 seed plants (SSCG; Supplementary Table 13). For each gene, amino acid sequences were aligned using MAFFT $^{57}$ v.7.402, and then DNA sequences were aligned according to the corresponding amino acid alignments using PAL2NAL ${ }^{58}$ v.14. For datasets SSCG-CDS and SSCG-Codon12, the maximum likelihood (ML) trees were inferred from concatenated gene sequences using IQ-TREE ${ }^{59}$ v.1.6.9, which automatically selected the best-fit substitution model using ModelFinder ${ }^{60}$ Bootstrap support was estimated using 1,000 replicates of the ultrafast bootstrap approximation $^{61}$ (-bb 1000 -m MFP). For coalescent-based analyses, gene trees were first estimated using IQ-TREE; the gene trees were then utilized by ASTRAL v.5.6.1 to infer species trees with quartet scores and posterior probabilities. Furthermore, SSCGs were identified using OrthoMCL ${ }^{36}$ v.2.0.9 (OSCG) with one more Gymnosperm (P. abies; Supplementary Table 13). Species trees were inferred from the datasets OSCG-CDS and OSCG-Codon 12 using concatenation and coalescent methods as described above. Finally, we extracted low-copy genes from 15 seed plants (LCG). Here, each gene was required to include at least 1 sequence from each of the 15 species and less than 5 homologous sequences per species. For the datasets LCG-CDS and LCG-Codon12, gene trees were first estimated using

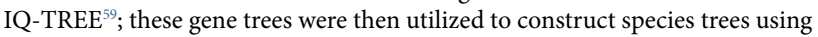
$\mathrm{STAG}^{37}$ v.1.0.0.

For plastid genes, the 72 CDS of protein-coding genes were extracted from 15 seed plants (Supplementary Table 14), and aligned using MAFFT and PAL2NAL as described above. The ML trees were inferred from concatenated gene sequences using RAxML ${ }^{62}$ v.7.2.8 with 100 bootstraps.

For expanded taxon sampling, sequence similarity was first assessed for all of the amino acid sequences from 213 species (211 angiosperms and 2 gymnosperms; Supplementary Table 15) using MMseqs $2^{63}$ with an $E$-value threshold of $1 \times 10^{-5}$, and then grouped using a Markov cluster algorithm ${ }^{64}$. Here, each gene was required to include sequences from more than 180 species. Next, 'mostly' single-copy orthologous genes (SCOG) were identified using a tree-based method ${ }^{65,66}$. Each gene was aligned using MAFFT and PAL2NAL as described above, and species trees were inferred from datasets SCOG-CDS and SCOG-Codon12 using ASTRAL.

Visualizations of gene-tree discordance. Gene trees were first converted to ultrametric trees using the R package Phybase ${ }^{67}$, and then superimposed using DensiTree $^{38}$ (Fig. 2c). Quartet frequencies of the internal branches in the species tree were calculated using ASTRAL ${ }^{33}$ with the parameter ' $-\mathrm{t}=2$ ' (Supplementary Figs. 17 and 19). Furthermore, the analysis of gene-tree compatibility was conducted using DiscoVista ${ }^{39}$ v.1.0. Here, a total of 15 species groups were considered, and 7 of which are identified in our species tree, including: (1) 12 Mesagiospermae, (2) 4 eudicots, (3) 3 magnollids, (4) 3 monocots, (5) Euryale and all Mesangiospermaes, (6) Ceratophyllaceae and eudicots, and (7) Magnoliids and (Ceratophyllaceae and eudicots). The other 8 species groups were: (1) basal angiosperms (that is, Ambrorella and Euryale), (2) Ambrorella and Mesangiospermae, (3) Ceratophyllaceae and magnoliids, (4) Ceratophyllaceae and monocots, (5) magnoliids and eudicots, (6) magnoliids and monocots, (7) eudicots and monocots, and (8) Ceratophyllaceae, magnoliids and monocots (Fig. 2e, Supplementary Fig. 18). Bootstrap support values of at least $75 \%$ were interpreted as highly supported ${ }^{68}$ (Fig. 2e).

Divergence time estimation. Divergence time was estimated for the dataset SSCGCDS using the program MCMCTree in the PAML ${ }^{53}$ package v.4.9h. After a burnin of 5,000,000 iterations, the MCMC process was performed 20,000 times with sample frequency of 5,000. Convergence was assessed using two independent runs. We used the following age constraints in our estimation procedure: the divergence between angiosperms and gymnosperms (330-289 Ma; http://www.timetree.org/), the crown group of angiosperms $(267-132.9 \mathrm{Ma})^{4}$, the crown group of monocots $(184-113 \mathrm{Ma})^{4}$ and the crown group of eudicots $(161-125 \mathrm{Ma})^{4}$.

Hybridization inference and ILS simulation. Hybridization was detected for the dataset OSCG-CDS using the maximum pseudolikelihood estimation of phylogenetic networks, as implemented in PhyloNetworks ${ }^{69}$ v.0.9.0. The maximum allowed number of hybridizations was set from $h \max =0$ to $h \max =10$, each with 100 runs. The ILS simulation was performed as previously described ${ }^{70}$. We simulated 200,000 gene trees under the multispecies coalescent model using the $\mathrm{R}$ function sim.coaltree.sp as implemented in the package Phybase $\mathrm{e}^{67} \mathrm{v} .1 .5$. The internal branch lengths of the ASTRAL tree were used for the simulation, and all terminal branches were set to 1 (as 1 allele was generated for each species). It should be noted that internal branch lengths (in coalescent units) in our simulation might have been overestimated, as the cause of gene tree heterogeneity was assumed to result from only ILS. Gene-tree quartet frequencies were calculated for simulated and empirical datasets, and the correlation test was performed using the cor.test function in $\mathrm{R}$.

Demographic inference. The pairwise sequentially Markovian coalescent (PSMC) model $^{71}$ v.0.6.4-r49 was used to infer the demographic history of seven species, that is, A. trichopoda (Amborellales), E. ferox (Nymphaeales), C. demersum (Ceratophyllales), C. kanehirae (magnoliids), L. chinense (magnoliids), $P$. equestris (monocots) and $V$. vinifera (eudicots). The genome of E. ferox showed very low heterozygosity (about $0.02 \%$; Supplementary Table 22 ) and, therefore, two individuals were included in the PSMC analysis ${ }^{72}$. For each species, wholegenome resequencing data (at least 30 -fold coverage) were obtained from NCBI (Supplementary Table 22). Reads were mapped to the assembled genome, and the consensus sequences were extracted. The analysis was performed using the following parameters: -N25 -t15 -r5 -p ' $4+25 \times 2+4+6$ '. Here, for A. trichopoda, $C$ kanehirae, L. chinense and $V$. vinifera, the generation time and mutation rate were obtained from previous studies ${ }^{7,19,20,73}$. For other three species (that is, E. ferox, C. demersum and P. equestris), the mutation rate was first estimated using $\mathrm{r}^{8} \mathrm{~s}^{74}$. Furthermore, as E. ferox is an annual species, the generation time was set to 1 . For perennial species, as the generation time is difficult to determine precisely ${ }^{75}$, we tested the generation time for both 3 and 5 years, and similar results were obtained.

Reporting Summary. Further information on research design is available in the Nature Research Reporting Summary linked to this article.

\section{Data availability}

All of the raw sequence reads used in this study have been deposited at NCBI under the BioProject accession numbers PRJNA552436 (E. ferox) and PRJNA552433 (C. demersum). The assemblies and annotations are available from the CoGe comparative genomics platform at https://genomevolution.org/CoGe/GenomeInfo. $\mathrm{pl}$ ?gid $=56574$ (E. fero $x$ chromosome assembly), https://genomevolution.org/CoGe/ GenomeInfo.pl?gid=56571 (E. ferox contig assembly), https://genomevolution. org/CoGe/GenomeInfo.pl?gid=56572 (C. demersum chromosome assembly) and https://genomevolution.org/CoGe/GenomeInfo.pl?gid=56569 (C. demersum contig assembly).

\section{Code availability}

The custom scripts have deposited in GitHub (https://github.com/ yongzhiyang2012/Euryale_ferox_and_Ceratophyllum_demersum_genome_ analysis).

Received: 26 September 2019; Accepted: 6 January 2020; Published online: 24 February 2020

\section{References}

1. Judd, W. S., Campbell, C. S., Kellogg, E. A., Stevens, P. F. \& Donoghue, M. J. Plant Systematics (Sinauer Sunderland, 2002).

2. Friedman, W. E. The meaning of Darwin's 'abominable mystery'. Am. J. Bot 96, 5-21 (2009).

3. Buggs, R. J. A. The deepening of Darwin's abominable mystery. Nat. Ecol. Evol. 1, 0169 (2017).

4. Li, H. T. et al. Origin of angiosperms and the puzzle of the Jurassic gap. Nat. Plants 5, 461-470 (2019).

5. Steemans, P. et al. Origin and radiation of the earliest vascular land plants. Science 324, 353 (2009).

6. The Plant List. The Plant List - A working list of all plant species. Royal Botanic Gardens, Kew and Missouri Botanical Garden (2019). Available online at http://www.theplantlist.org/ (last retrieved $20 \mathrm{Aug} 2019$ ).

7. Amborella Genome Project. The Amborella genome and the evolution of flowering plants. Science 342, 1241089 (2013).

8. Qiu, Y.-L. et al. The earliest angiosperms: evidence from mitochondrial, plastid and nuclear genomes. Nature 402, 404-407 (1999).

9. Drinnan, A. N., Crane, P. R. \& Hoot, S. B. in Early Evolution of Flowers Supplement 8, Vol. 8 (eds Endress, P. K. \& Friis, E. M.) 93-122 (Springer, 1994).

10. Cronquist, A. \& Takhtadzhian, A. L. An Integrated System of Classification of Flowering Plants (Columbia Univ. Press, 1981).

11. Friis, E. M., Pedersen, K. R. \& Crane, P. R. Diversity in obscurity: fossil flowers and the early history of angiosperms. Proc. R. Soc. B 365, 369-382 (2010) 
12. Dilcher, D. L. \& Wang, H. An early cretaceous fruit with affinities to Ceratophyllaceae. Am. J. Bot. 96, 2256-2269 (2009).

13. Chase, M. W. et al. An update of the Angiosperm Phylogeny Group classification for the orders and families of flowering plants: APG IV. Bot. J. Linn. Soc. 181, 1-20 (2016).

14. Endress, P. K. \& Doyle, J. A. Reconstructing the ancestral angiosperm flower and its initial specializations. Am. J. Bot. 96, 22-66 (2009).

15. Moore, M. J., Bell, C. D., Soltis, P. S. \& Soltis, D. E. Using plastid genomescale data to resolve enigmatic relationships among basal angiosperms. Proc. Natl Acad. Sci. USA 104, 19363-19368 (2007).

16. Yin-Long, Q. et al. Angiosperm phylogeny inferred from sequences of four mitochondrial genes. J. Sys. Evol. 48, 391-425 (2010).

17. Zhang, N., Zeng, L., Shan, H. \& Ma, H. Highly conserved low-copy nuclear genes as effective markers for phylogenetic analyses in angiosperms. $N$. Phytol. 195, 923-937 (2012).

18. Zeng, L. et al. Resolution of deep angiosperm phylogeny using conserved nuclear genes and estimates of early divergence times. Nat. Commun. 5 , 4956 (2014).

19. Chaw, S.-M. et al. Stout camphor tree genome fills gaps in understanding of flowering plant genome evolution. Nat. Plants 5, 63-73 (2019).

20. Chen, J. et al. Liriodendron genome sheds light on angiosperm phylogeny and species-pair differentiation. Nat. Plants 5, 18-25 (2019).

21. Rendon-Anaya, M. et al. The avocado genome informs deep angiosperm phylogeny, highlights introgressive hybridization, and reveals pathogeninfluenced gene space adaptation. Proc. Natl Acad. Sci. USA 116, 17081-17089 (2019)

22. Soltis, D. E. \& Soltis, P. S. Nuclear genomes of two magnoliids. Nat. Plants 5 , 6-7 (2019).

23. Wickett, N. J. et al. Phylotranscriptomic analysis of the origin and early diversification of land plants. Proc. Natl Acad. Sci. USA 111, E4859-E4868 (2014).

24. Sun, M. et al. Deep phylogenetic incongruence in the angiosperm clade Rosidae. Mol. Phylogenet. Evol. 83, 156-166 (2015).

25 . Koren, S. et al. Canu: scalable and accurate long-read assembly via adaptive $k$-mer weighting and repeat separation. Genome Res. 27, 722-736 (2017).

26. Walker, B. J. et al. Pilon: an integrated tool for comprehensive microbial variant detection and genome assembly improvement. PLoS ONE 9, e112963 (2014).

27. Burton, J. N. et al. Chromosome-scale scaffolding of de novo genome assemblies based on chromatin interactions. Nat. Biotechnol. 31, 1119-1125 (2013).

28. Rice, A. et al. The Chromosome Counts Database (CCDB) - a community resource of plant chromosome numbers. N. Phytol. 206, 19-26 (2015)

29. Wang, X. et al. Statistical inference of chromosomal homology based on gene colinearity and applications to Arabidopsis and rice. BMC Bioinform. 7 , 447 (2006)

30. Estep, M. C. et al. Allopolyploidy, diversification, and the Miocene grassland expansion. Proc. Natl Acad. Sci. USA 111, 15149-15154 (2014).

31. Cai, L. et al. Widespread ancient whole-genome duplications in Malpighiales coincide with Eocene global climatic upheaval. N. Phytol. 221, 565-576 (2019).

32. Cosentino, S. \& Iwasaki, W. SonicParanoid: fast, accurate and easy orthology inference. Bioinformatics 35, 149-151 (2019).

33. Zhang, C., Rabiee, M., Sayyari, E. \& Mirarab, S. ASTRAL-III: polynomial time species tree reconstruction from partially resolved gene trees. $B M C$ Bioinform. 19, 153 (2018).

34. Moore, M. J. et al. Phylogenetic analysis of the plastid inverted repeat for 244 species: insights into deeper-level angiosperm relationships from a long, slowly evolving sequence region. Int. J. Plant Sci. 172, 541-558 (2011).

35. One Thousand Plant Transcriptomes Initiative One thousand plant transcriptomes and the phylogenomics of green plants. Nature $\mathbf{5 7 4}$ 679-685 (2019).

36. Li, L., Stoeckert, C. J. Jr \& Roos, D. S. OrthoMCL: identification of ortholog groups for eukaryotic genomes. Genome Res. 13, 2178-2189 (2003).

37. Emms, D. \& Kelly, S. STAG: species tree inference from all genes. Preprint at https://doi.org/10.1101/267914 (2018).

38. Bouckaert, R. R. DensiTree: making sense of sets of phylogenetic trees. Bioinformatics 26, 1372-1373 (2010).

39. Sayyari, E., Whitfield, J. B. \& Mirarab, S. DiscoVista: interpretable visualizations of gene tree discordance. Mol. Phylogenet. Evol. 122, 110-115 (2018).

40. Rannala, B. \& Yang, Z. H. Bayes estimation of species divergence times and ancestral population sizes using DNA sequences from multiple loci. Genetics 164, 1645-1656 (2003)

41. Hillis, D. M., Pollock, D. D., McGuire, J. A. \& Zwickl, D. J. Is sparse taxon sampling a problem for phylogenetic inference? Syst. Biol. 52, 124-126 (2003).

42. Tel-Zur, N., Abbo, S., Myslabodski, D. \& Mizrahi, Y. Modified CTAB procedure for DNA isolation from epiphytic cacti of the genera Hylocereus and Selenicereus (Cactaceae). Plant Mol. Biol. Rep. 17, 249-254 (1999).
43. Luo, R. et al. SOAPdenovo2: an empirically improved memory-efficient short-read de novo assembler. Gigascience 1, 18 (2012).

44. Shi, J. et al. Chromosome conformation capture resolved near complete genome assembly of broomcorn millet. Nat. Commun. 10, 464 (2019).

45. Servant, N. et al. HiC-Pro: an optimized and flexible pipeline for Hi-C data processing. Genome Biol. 16, 259 (2015).

46. Grabherr, M. G. et al. Full-length transcriptome assembly from RNA-Seq data without a reference genome. Nat. Biotechnol. 29, 644-652 (2011).

47. Tarailo-Graovac, M. \& Chen, N. Using RepeatMasker to identify repetitive elements in genomic sequences. Curr. Protoc. Bioinform. 25, 4.10.1-4.10.14 (2009).

48. Haas, B. J. et al. Improving the Arabidopsis genome annotation using maximal transcript alignment assemblies. Nucleic Acids Res. 31, 5654-5666 (2003)

49. Stanke, M., Diekhans, M., Baertsch, R. \& Haussler, D. Using native and syntenically mapped cDNA alignments to improve de novo gene finding. Bioinformatics 24, 637-644 (2008)

50. Jones, P. et al. InterProScan 5: genome-scale protein function classification. Bioinformatics 30, 1236-1240 (2014)

51. Tang, H. B. et al. Unraveling ancient hexaploidy through multiply-aligned angiosperm gene maps. Genome Res. 18, 1944-1954 (2008).

52. Nei, M. \& Gojobori, T. Simple methods for estimating the numbers of synonymous and nonsynonymous nucleotide substitutions. Mol. Biol. Evol. 3 418-426 (1986)

53. Yang, Z. PAML 4: phylogenetic analysis by maximum likelihood. Mol. Biol. Evol. 24, 1586-1591 (2007).

54. Wang, X. et al. Genome alignment spanning major Poaceae lineages reveals heterogeneous evolutionary rates and alters inferred dates for key evolutionary events. Mol. Plant 8, 885-898 (2015).

55. Vekemans, D. et al. Gamma paleohexaploidy in the stem lineage of core eudicots: significance for MADS-box gene and species diversification. Mol. Biol. Evol. 29, 3793-3806 (2012).

56. Jiao, Y. N. et al. Ancestral polyploidy in seed plants and angiosperms. Nature 473, 97-100 (2011).

57. Katoh, K., Misawa, K., Kuma, K. \& Miyata, T. MAFFT: a novel method for rapid multiple sequence alignment based on fast Fourier transform. Nucleic Acids Res. 30, 3059-3066 (2002).

58. Suyama, M., Torrents, D. \& Bork, P. PAL2NAL: robust conversion of protein sequence alignments into the corresponding codon alignments. Nucleic Acids Res. 34, W609-W612 (2006).

59. Nguyen, L. T., Schmidt, H. A., von Haeseler, A. \& Minh, B. Q. IQ-TREE: a fast and effective stochastic algorithm for estimating maximum-likelihood phylogenies. Mol. Biol. Evol. 32, 268-274 (2015).

60. Kalyaanamoorthy, S., Minh, B. Q., Wong, T. K. F., von Haeseler, A. \& Jermiin L. S. ModelFinder: fast model selection for accurate phylogenetic estimates. Nat. Methods 14, 587-589 (2017).

61. Hoang, D. T., Chernomor, O., von Haeseler, A., Minh, B. Q. \& Vinh, L. S. UFBoot2: improving the ultrafast bootstrap approximation. Mol. Biol. Evol. 35, 518-522 (2018).

62. Stamatakis, A. RAxML version 8: a tool for phylogenetic analysis and post-analysis of large phylogenies. Bioinformatics 30, 1312-1313 (2014).

63. Steinegger, M. \& Soding, J. MMseqs2 enables sensitive protein sequence searching for the analysis of massive data sets. Nat. Biotechnol. 35 , 1026-1028 (2017)

64. Enright, A. J., Van Dongen, S. \& Ouzounis, C. A. An efficient algorithm for large-scale detection of protein families. Nucleic Acids Res. 30, 1575-1584 (2002)

65. Yang, Y. \& Smith, S. A. Orthology inference in nonmodel organisms using transcriptomes and low-coverage genomes: improving accuracy and matrix occupancy for phylogenomics. Mol. Biol. Evol. 31, 3081-3092 (2014).

66. De Smet, R. et al. Convergent gene loss following gene and genome duplications creates single-copy families in flowering plants. Proc. Natl Acad. Sci. USA 110, 2898-2903 (2013).

67. Liu, L. \& Yu, L. Phybase: an R package for species tree analysis. Bioinformatics 26, 962-963 (2010).

68. Jarvis, E. D. et al. Whole-genome analyses resolve early branches in the tree of life of modern birds. Science 346, 1320-1331 (2014).

69. Solis-Lemus, C., Bastide, P. \& Ane, C. PhyloNetworks: a package for phylogenetic networks. Mol. Biol. Evol. 34, 3292-3298 (2017).

70. Wang, K. et al. Incomplete lineage sorting rather than hybridization explains the inconsistent phylogeny of the wisent. Commun. Biol. 1, 169 (2018).

71. Li, H. \& Durbin, R. Inference of human population history from individual whole-genome sequences. Nature 475, 493-496 (2011).

72. Thomas, C. G. et al. Full-genome evolutionary histories of selfing, splitting, and selection in Caenorhabditis. Genome Res. 25, 667-678 (2015).

73. Zhou, Y., Massonnet, M., Sanjak, J. S., Cantu, D. \& Gaut, B. S. Evolutionary genomics of grape (Vitis vinifera ssp. vinifera) domestication. Proc. Natl Acad. Sci. USA 114, 11715-11720 (2017). 
74. Sanderson, M. J. r8s: inferring absolute rates of molecular evolution and divergence times in the absence of a molecular clock. Bioinformatics 19, 301-302 (2003).

75. Petit, R. J. \& Hampe, A. Some evolutionary consequences of being a tree. Annu. Rev. Ecol. Evol. Syst. 37, 187-214 (2006).

\section{Acknowledgements}

This work was supported equally by the Strategic Priority Research Program of Chinese Academy of Sciences (XDB31010300) and the National Key Research and Development Program of China (2017YFC0505203), and further by the National Natural Science Foundation of China (grant numbers 31590821, 91731301 and 31561123001), the Fundamental Research Funds for the Central Universities 2018CDDY-S02-SCU and SCU2019D013, and National High-Level Talents Special Support Plans.

\section{Author contributions}

J.L. was the leader of this study. J.L., Z.X., X.W. and C.C.D. designed the experiments and coordinated the project. L.Z., D.W. and Q.W. performed field work and collected samples. Y.Y., L.L., D.W. and D.R. performed the assembly of the two genomes. Y.Y., L.L., T.M. and D.R. carried out the repeat and gene annotations. X.W., P.S., F.M., B.J., L.S., M.L. and Z.Q. performed the polyploidization analysis. Y.Y., L.L., D.R., Y.L. and X.S. carried out the gene family analysis and the phylogenomic analysis. Y.Y. and Y.L. performed the PSMC analysis. Y.Y., J.L., Z.X., C.C.D. and X.W. wrote and edited most of the manuscript. All of the authors read and approved the final manuscript.

\section{Competing interests}

The authors declare no competing interests.

\section{Additional information}

Supplementary information is available for this paper at https://doi.org/10.1038/ s41477-020-0594-6.

Correspondence and requests for materials should be addressed to Z.X., X.W., C.C.D. or J.L.

Peer review information Nature Plants thanks Victor Albert and the other, anonymous, reviewer(s) for their contribution to the peer review of this work.

Reprints and permissions information is available at www.nature.com/reprints.

Publisher's note Springer Nature remains neutral with regard to jurisdictional claims in published maps and institutional affiliations.

(c) (i) Open Access This article is licensed under a Creative Commons

Attribution 4.0 International License, which permits use, sharing, adaptation, distribution and reproduction in any medium or format, as long as you give appropriate credit to the original author(s) and the source, provide a link to the Creative Commons license, and indicate if changes were made. The images or other third party material in this article are included in the article's Creative Commons license, unless indicated otherwise in a credit line to the material. If material is not included in the article's Creative Commons license and your intended use is not permitted by statutory regulation or exceeds the permitted use, you will need to obtain permission directly from the copyright holder. To view a copy of this license, visit http://creativecommons.org/licenses/by/4.0/.

(C) The Author(s) 2020 


\section{natureresearch}

Corresponding author(s): Jianquan Liu

Last updated by author(s): Dec 25, 2019

\section{Reporting Summary}

Nature Research wishes to improve the reproducibility of the work that we publish. This form provides structure for consistency and transparency in reporting. For further information on Nature Research policies, see Authors \& Referees and the Editorial Policy Checklist.

\section{Statistics}

For all statistical analyses, confirm that the following items are present in the figure legend, table legend, main text, or Methods section.

n/a Confirmed

\ The exact sample size $(n)$ for each experimental group/condition, given as a discrete number and unit of measurement

$\square$ A statement on whether measurements were taken from distinct samples or whether the same sample was measured repeatedly

$\square$ The statistical test(s) used AND whether they are one- or two-sided

Only common tests should be described solely by name; describe more complex techniques in the Methods section.

Х $\square$ A description of all covariates tested

$\square \bigotimes$ A description of any assumptions or corrections, such as tests of normality and adjustment for multiple comparisons

A full description of the statistical parameters including central tendency (e.g. means) or other basic estimates (e.g. regression coefficient) AND variation (e.g. standard deviation) or associated estimates of uncertainty (e.g. confidence intervals)

For null hypothesis testing, the test statistic (e.g. $F, t, r$ ) with confidence intervals, effect sizes, degrees of freedom and $P$ value noted Give $P$ values as exact values whenever suitable.

\ For Bayesian analysis, information on the choice of priors and Markov chain Monte Carlo settings

Х $\square$ For hierarchical and complex designs, identification of the appropriate level for tests and full reporting of outcomes

$\square$ Estimates of effect sizes (e.g. Cohen's $d$, Pearson's $r$ ), indicating how they were calculated

\section{Our web collection on statistics for biologists contains articles on many of the points above.}

\section{Software and code}

Policy information about availability of computer code

Data collection

Data analysis

\section{No software was used to collect the data.}

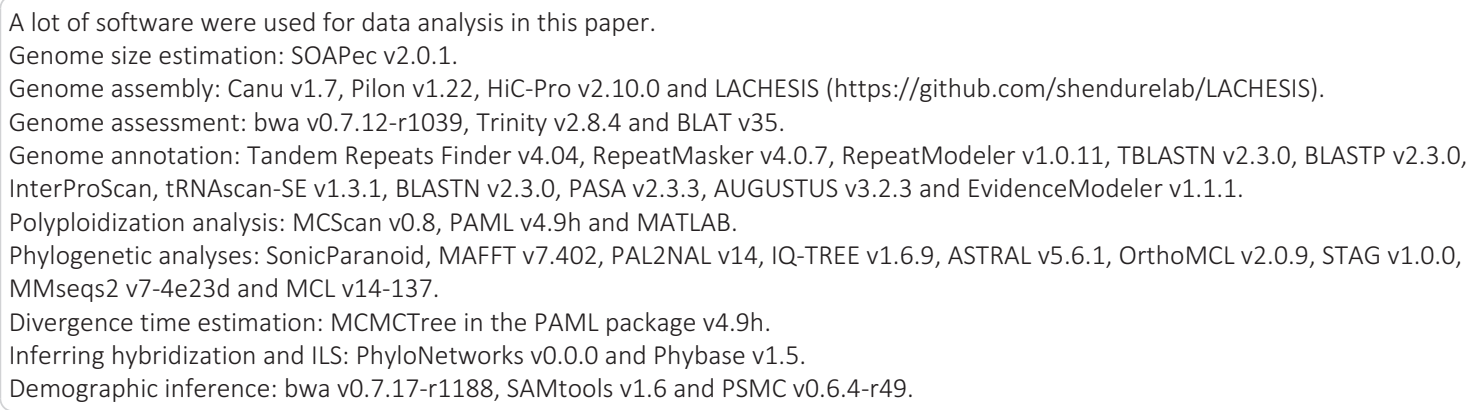

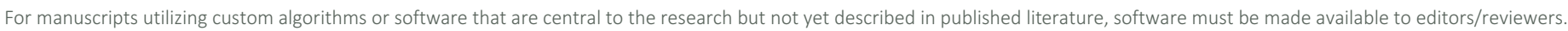
We strongly encourage code deposition in a community repository (e.g. GitHub). See the Nature Research guidelines for submitting code \& software for further information. 
Policy information about availability of data

All manuscripts must include a data availability statement. This statement should provide the following information, where applicable:

- Accession codes, unique identifiers, or web links for publicly available datasets

- A list of figures that have associated raw data

- A description of any restrictions on data availability

All the raw sequence reads used in this study have been deposited in NCBI under the BioProject accession numbers PRJNA552436 (E. ferox) and PRJNA552433 (C. demersum). The assemblies and annotations are available from the CoGe comparative genomics platform: https://genomevolution.org/CoGe/Genomelnfo.pl? gid=56574 (E. ferox chromosome assembly), https://genomevolution.org/CoGe/Genomelnfo.pl?gid=56571 (E. ferox contig assembly), https://genomevolution.org/ CoGe/Genomelnfo.pl?gid=56572 (C. demersum chromosome assembly) and https://genomevolution.org/CoGe/Genomelnfo.pl?gid=56569 (C. demersum contig assembly). The custom scripts have deposited in GitHub (https://github.com/yongzhiyang2012/Euryale_ferox_and_Ceratophyllum_demersum_genome_analysis).

\section{Field-specific reporting}

Please select the one below that is the best fit for your research. If you are not sure, read the appropriate sections before making your selection.

Х Life sciences

Behavioural \& social sciences

Ecological, evolutionary \& environmental sciences

For a reference copy of the document with all sections, see nature.com/documents/nr-reporting-summary-flat.pdf

\section{Life sciences study design}

All studies must disclose on these points even when the disclosure is negative.

Sample size One Prickly waterlily and one rigid hornwort individuals were selected containing the sufficiently fresh samples to the genome sequencing. As the very low heterozygosity detected within Prickly waterlily, another individual was needed and sequenced for the PSMC analysis.

Data exclusions For the long reads, we have removed the reads with a mean quality score $<7$. For the short Illumina reads, the following criteria were performed to filter the low quality reads: (i) containing more than $5 \%$ unidentified nucleotides, (ii) more than $65 \%$ of bases with a Phred quality score $<7$, and (iii) more than 10 bp adapter sequences (allowing 2 bp mismatches)

Replication No replication in this manuscript.

Randomization No randomization in this manuscript as the genome assembly, annotation and comparison no needed randomization. For the phylogeny analysis we used all the identified single copy genes or low copy genes, not underling a randomization.

Blinding

The two genome were sequenced and assembled with no blinding as data were not allocated into groups.

\section{Reporting for specific materials, systems and methods}

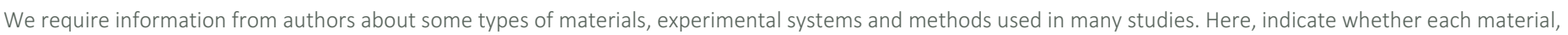

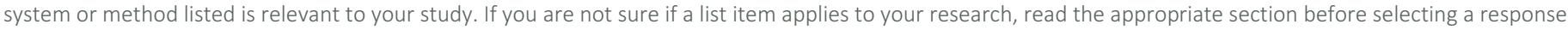

\begin{tabular}{|c|c|c|c|}
\hline \multicolumn{2}{|r|}{ Materials \& experimental systems } & \multicolumn{2}{|c|}{ Methods } \\
\hline$n / a$ & Involved in the study & $\mathrm{n} / \mathrm{a}$ & Involved in the study \\
\hline$\bigotimes$ & $\square$ Antibodies & Х & $\square$ ChIP-seq \\
\hline$\bigotimes$ & $\square$ Eukaryotic cell lines & Х & $\square$ Flow cytometry \\
\hline Х & $\square$ Palaeontology & Х & $\square$ MRI-based neuroimaging \\
\hline$\bigotimes$ & $\square$ Animals and other organisms & & \\
\hline$\bigotimes$ & $\square$ Human research participants & & \\
\hline 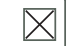 & $\square$ Clinical data & & \\
\hline
\end{tabular}

